\title{
ANALYSIS OF WALL SHAPE IN INDOOR AIR CIRCULATION BY THE FINITE ELEMENT METHOD
}

\author{
P. R. C. Drach, \\ and J. Karam F. \\ ABSTRACT \\ The use of computational models in built environments comes from the \\ need to deal with situations as close as possible to the reality and also to \\ study functional spaces that could be able to provide, for example, thermal \\ comfort. In this work we analyze some cases of indoor air circulation in \\ Laboratório Nacional de Computação \\ built environments through a mixed stabilized finite element method, \\ applied to the Navier-Stokes equations in velocity and pressure variables. \\ The implemented numerical method ensures stability for the internal \\ constraint imposed by the velocity field, and accommodates moderate to \\ large advective effects. The obtained internal wind field allows the choice \\ of wall shapes that increase or not the ventilation and can alter its \\ distribution, allowing in this way a better adequacy of the built environment \\ for the climate needs and its objectives. \\ pdrach@Incc.br \\ jkfi@lncc.br \\ Keywords: built environment, air circulation, indoor thermal comfort, finite \\ element method, wall shape.
}

\section{INTRODUCTION}

Nowadays, environmental needs of working and living spaces are changing very fast; we need to save energy, obtain low pollution and use appropriate materials if we want to produce inexpensive and efficient buildings. The goal is to build a place to protect, being at the same time functional and, therefore, with acoustic, luminic, thermal and hygienic cares.

In the specific case of the climate, we need to maintain internal heat in very cold places and to promote a better ventilation in hot climates, adopting in many circumstances mechanical ventilation.

In naturally ventilated environment, thermal conditions are directly related to the air circulation and the characteristics of the built environment. The air circulation around the human body, as it is known, provides a heat transfer from the skin to the environment, in case of cooled air. According to Szokolay (1999), when the skin is in contact with the air at $1.5 \mathrm{~m} / \mathrm{s}$, thermal neutrality may be increased up to $6 \mathrm{~K}$. There is still the fact that people can support better the same level of temperature in naturally ventilated environment rather than when exposed to air-conditioned equipments, (Bragger and Dear, 2000).

The study of natural indoor air circulation by computational methods can help to evaluate and plan better built environment in terms of comfort, health and functionality.

In this work, we focus our attention in the circulation aspects that could influence the thermal comfort in a specific built environment by starting with the original project and analyzing three alternative modifications of wall shape combined with the plans of the internal doors. It was considered a two dimensional computational approach, that can be used in 3D-by-layers schemes and can be extended to a 3D full approximation, once the mathematical formulation has no restriction to any dimension.

The wind fields have been obtained by computational simulations performed with the use of a mixed stabilized finite element method, like PetrovGalerkin (Brooks and Hughes, 1982), on the NavierStokes equations in primitives variables. Numerical results are presented and some conclusions are done.

\section{MATHEMATICAL FORMULATION}

Air circulation can be modelled through mass and momentum conservation equations in velocity and pressure. The momentum equations in this case are the Navier-Stokes equations and assuming incompressibility the mathematical formulation for the problem can be written as:

Find $\mathbf{u}$ and p satisfying the following system,

$\operatorname{div}(\mathbf{u})=0$, in $\Omega \times[0, \mathrm{~T}]$,

$\rho \frac{\partial \mathbf{u}}{\partial \mathrm{t}}+\rho(\nabla \mathbf{u}) \mathbf{u}-2 \mu \operatorname{div} \varepsilon(\mathbf{u})+\nabla \mathrm{p}=0$,

in $\Omega \times[0, T]$

With initial and boundary conditions,

$$
\begin{aligned}
& \nabla(\mathbf{u}) . \mathbf{1} \\
& \text { and } \mathbf{u}(\mathbf{x}, 0)=0 \text { on } \Gamma_{v} \times[0, \mathrm{~T}], \mathbf{u}(\mathbf{x}, \mathrm{t})=\overline{\mathbf{u}}(\mathbf{x}, \mathrm{t}) \text { on } \Gamma_{u} \times[0, \mathrm{~T}]
\end{aligned}
$$

where: $\mathbf{u}=\mathbf{u}(\mathbf{x}, t)$ is the velocity vector, $\mathrm{p}=(\mathbf{x}, t)$ is the pressure, $\mu$ is the viscosity, $\rho$ is the density, $\underset{\mathbf{n}}{\mathbf{r}}$ is the normal vector, $\varepsilon(\mathbf{u})=1 / 2\left(\nabla \mathbf{u}+\nabla \mathbf{u}^{T}\right), \Omega$ is the bounded domain with boundary $\Gamma=\Gamma_{v} \mathrm{U} \Gamma_{u}$ and the time $t \in[0, \mathrm{~T}]$. 


\section{FINITE ELEMENT METHOD}

Two basic difficulties come from the equation system Eq. 1 - Eq. 2: the satisfaction of the incompressibility constraint that may generate locking of the velocity field or spurious pressures when classical formulations are used; and the presence of the nonlinear term in the Navier-Stokes equations that can cause numerical instabilities when advection effects are dominant. To ensure numerical stability and preserve the mathematical consistence, some stabilized methods have been created as, for example, Consistent Approximate Upwind (CAU) of Galeão and do Carmo (1991), and the Streamline Upwind Petrov-Galerkin (SUPG), Brooks and Hughes (1982). Both of them are based on the modification of the classical Galerkin method through the addition of residue perturbation terms with stabilizing parameters.

Here, a Petrov-Galerkin type method has been implemented and applied to analyzed indoor air circulation cases ensuring stability for dominant advection and for the internal constraint, (Karam F. and Loula, 1992)

Being $L^{2}$ and $H^{1}$ the usual Hilbert spaces and $R_{l}^{h}$ the Lagrange polynomial space of the degree $l$ and class $C^{0}$. Then, defining the following approximation spaces

$V_{h}=\left\{\mathbf{u}_{h} \in\left(H_{0}^{1}(\Omega) \cap R_{l}^{h}(\Omega)\right)^{2}, \mathbf{u}_{h}(\mathbf{x}, \mathrm{t})=\overline{\mathbf{u}}_{h}(\mathbf{x}, \mathrm{t})\right.$ in $\left.\Gamma_{u}\right\} \subset\left(H^{1}(\Omega)\right)^{2}$

$V_{h}^{0}=\left\{\mathbf{v}_{h} \in\left(H_{0}^{1}(\Omega) \cap R_{l}^{h}(\Omega)\right)^{2}, \mathbf{v}_{h}(\mathbf{x}, \mathrm{t})=0\right.$ in $\left.\Gamma_{u}\right\} \subset\left(H^{1}(\Omega)\right)^{2}$

$P^{h}=\left\{p_{h} \in\left(L^{2}(\Omega) \cap R_{l}^{h}(\Omega)\right) ; \int_{\Omega} p_{h} \partial \Omega=0\right\} \subset\left(L^{2}(\Omega)\right)$

with the usual norm

$\|\mathbf{u}\|_{1}^{2}=\|\mathbf{u}\|_{0}^{2}+\|\nabla \mathbf{u}\|_{0}^{2}$ of $H^{1}$ and $\|p\|=\|p\|_{0}$ of $L^{2}$.

The wind field can be determined by solving the following formulation:

Find $\left\{\mathbf{u}^{h}, p^{h}\right\} \in V^{h} \times P^{h}$ satisfying the following system

$B\left(\mathbf{u}_{h}, p_{h} ; \mathbf{v}_{h}, q_{h}\right)=0, \forall\left(\mathbf{v}_{h}, q_{h}\right) \in V_{h} \times P_{h}$

where:

$\mathrm{B}\left(\mathbf{u}_{h}, \mathrm{p}_{h} ; \mathbf{v}_{h}, \mathrm{q}_{h}\right)=\left(\frac{\partial \mathbf{u}_{h}}{\partial t}, \mathbf{v}_{h}\right)+\left(\left(\nabla \mathbf{u}_{h}\right) \mathbf{a}_{h}, \mathbf{v}_{h}\right)+$

$2 v\left(\varepsilon\left(\mathbf{u}_{h}\right), \varepsilon\left(\mathbf{v}_{h}\right)\right)-\left(p_{h}, \operatorname{div}\left(\mathbf{v}_{h}\right)\right)+\left(q_{h}, \operatorname{div}\left(\mathbf{u}_{h}\right)\right)+$

$\left(\operatorname{div}\left(\mathbf{u}_{h}\right), \delta_{2} \operatorname{div}\left(\mathbf{v}_{h}\right)\right)+$

$\delta_{1} \sum_{e=1}^{N e l}\left(\frac{\partial \mathbf{u}_{h}}{\partial t}+\left(\nabla \mathbf{u}_{h}\right) \mathbf{a}_{h}-2 v \operatorname{div} \varepsilon\left(\mathbf{u}_{h}\right)+\nabla \mathrm{p}_{h}\right.$,

$\left.\left(\left(\nabla \mathbf{v}_{h}\right) \mathbf{a}_{h}-2 v \operatorname{div} \varepsilon\left(\mathbf{v}_{h}\right)+\nabla q_{h}\right)\right)_{\mathrm{h}}+\gamma\left(\mathrm{p}_{h}, q_{h}\right)$,

$\forall \mathbf{v}_{\mathrm{h}} \in V_{h}^{0}$ e $q_{h} \in P_{h}$. with $\gamma \quad<1$ and $\delta_{1}$ and $\delta_{2} \quad$ stabilized parameters suggested by (Franca and Frey, 1992 ).

The time discretization has been done by the usual backward Euler finite difference.

\section{ORIGINAL PROJECT AND ITS MODIFICATIONS}

The case analyzed here consists of three prototypes of a residence module: the original plan that is drawn in Fig. 1(a) and the proposed modifications that are illustrated in Fig. 1(b) and Fig. 2. These modifications have been done to provide an improved plan for the air circulation inside the residence, by simply altering the shape of one of the walls, trying an easy and economical alternative. In Fig 1(a) the arrows indicate the direction of the outside wind, providing the boundary conditions. The adopted absolute value of this wind was $1 \mathrm{~m} / \mathrm{s}$, according to the Beaufort Scale, used in time forecast, corresponding to a breeze.

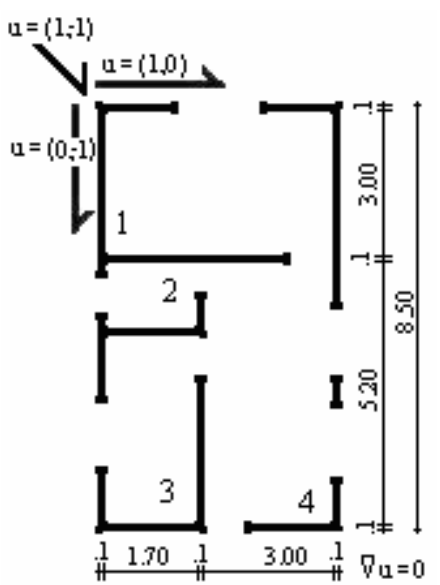

(a) Plan 1 - original plan.

Figure 1: Sketches of the first two plans.

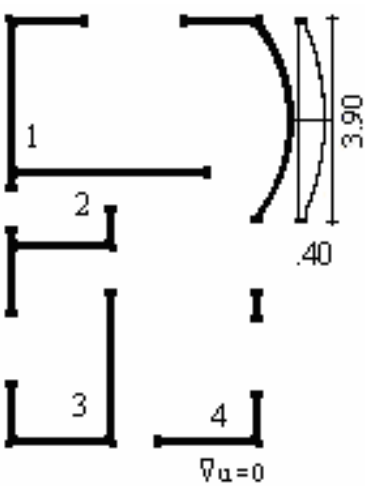

(a) Plan 3 - concave wall with detail.

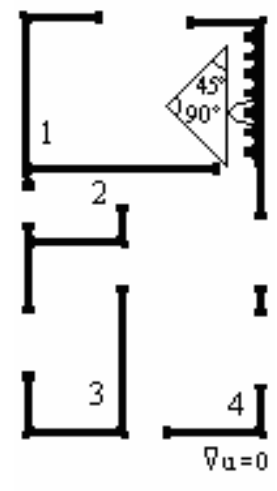

(b) Plan 2 - corrugated wall with detail.
Figure 2: Sketches of the last two plans. 


\section{SIMULATIONS AND RESULTS}

For the four geometry configuration cases, simulations have been done for each of the three wind directions indicated in Fig. 1(a), with a total of twelve simulation cases. The dimensions of the rooms are indicated in Fig. 1(a). The modifications of one of the walls are marked in Fig. 1(b) with a corrugated wall, Fig. 2(a) with a concave wall and Fig. 2(b) with a convex wall. Meshes and results for each case are presented in Figs. 4 -19 for the plan areas. For all the cases the whole mesh comprises an area of $20 \times 23.5 \mathrm{~m}^{2}$, see Fig. 3, bigger than the area of the plans in order to impose the boundary conditions on its border and to leave unknown the velocities at the entrances that are determined by the solution of the problem. Results are presented in terms of velocity vector fields in $\mathrm{m} / \mathrm{s}$, contour fill of $|\mathbf{u}|$ and contour lines of $|\mathbf{u}|$. Color scale is the same for all the results presented.

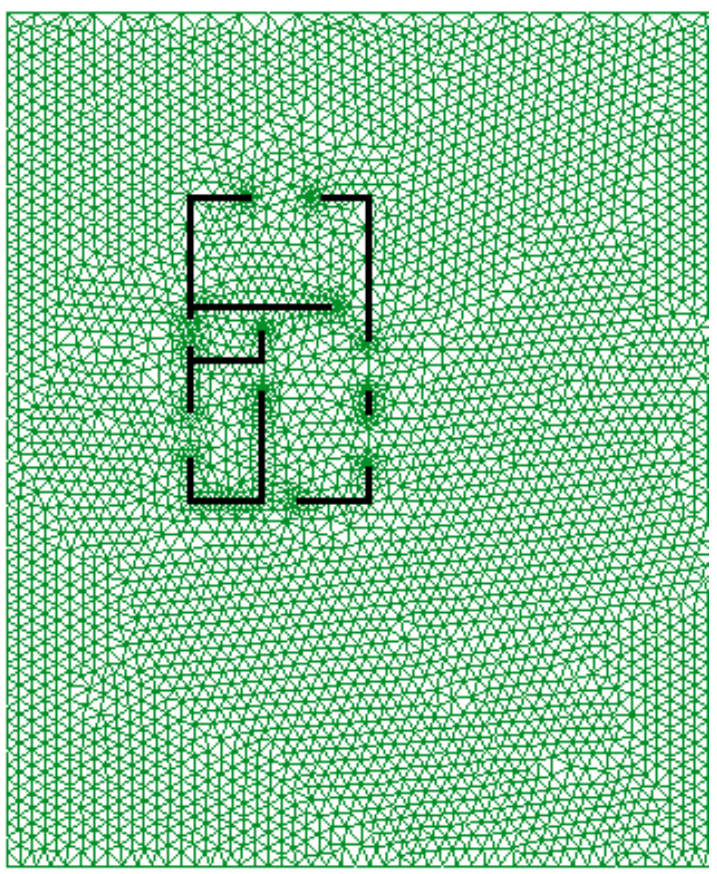

Figure 3: Whole mesh.

\section{Results for configuration Case 1.}

Figures 4, 5, 6 and 7 present internal velocity fields for the three given outside wind directions for Plan 1. The mesh has 7050 triangular elements and 3723 nodes as in Fig. 4(a).

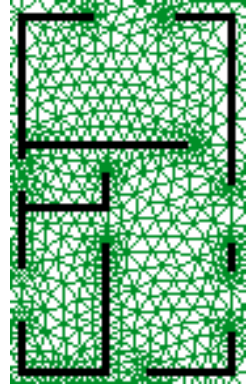

(a) Mesh detail.

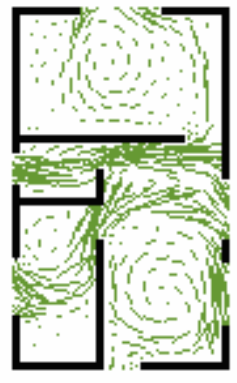

(b)Velocity field.
Figure 4: Mesh detail and wind field for Plan 1 and $\mathbf{u}=(1,0)$.

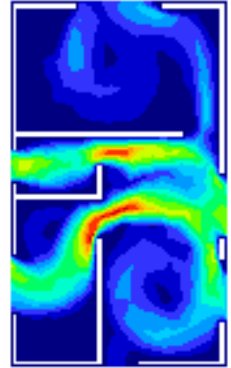

(a) Contour fill.

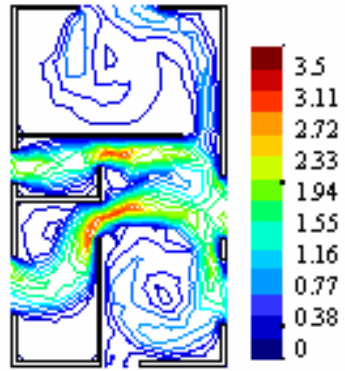

(b) Contour lines.
Figure 5: Contour fill and contour lines of $|\mathbf{u}|$ for Plan 1 and $\mathbf{u}=(1,0)$.
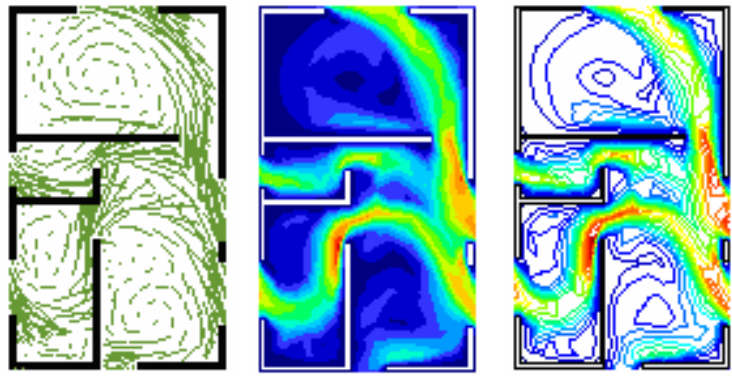

(a)Velocity field. (b)Contour fill. (c)Contour lines. Figure 6: Velocity field, contour fill and contour lines of $|\mathbf{u}|$ for Plan 1 and $\mathbf{u}=(1,-1)$.
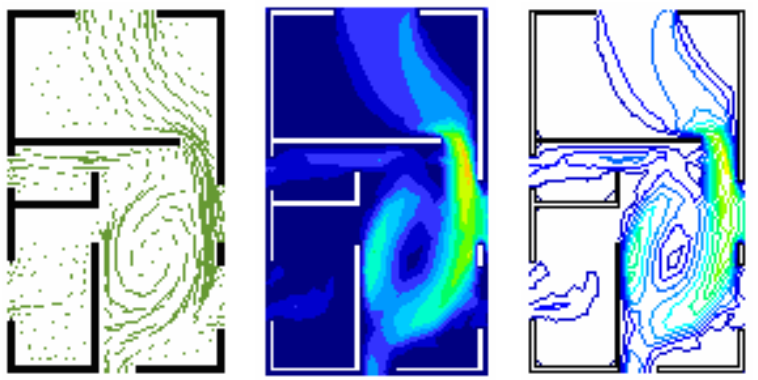

(a)Velocity field. (b)Contour fill. (c)Contour lines.

Figure 7: Velocity field, contour fill and contour lines of $|\mathbf{u}|$ for Plan 1 and $\mathbf{u}=(0,-1)$. 


\section{Results for configuration Case 2.}

Figures 8, 9, 10 and 11 present internal circulation results for the three given outside wind for Plan 2. The mesh has 7240 triangular elements and 3856 nodes as in Fig. 8(a). Comparing with the results obtained for Plan 1, the modified Plan 2 gave rise a stronger internal wind field with the circulation reaching a wider area.

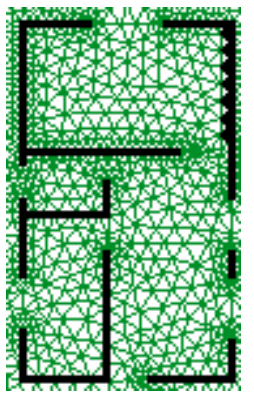

(a) Mesh detail.

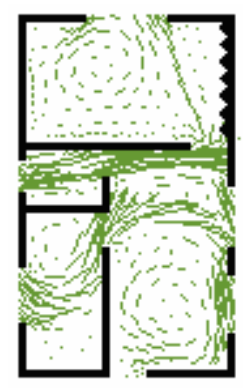

(b) Velocity field.
Figure 8: Mesh detail and wind field for Plan 2 and $\mathbf{u}=(1,0)$.

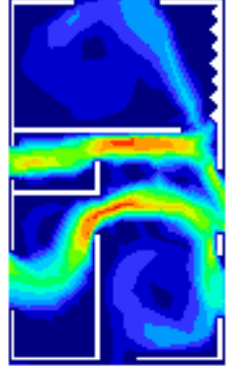

(a) Contour fill.

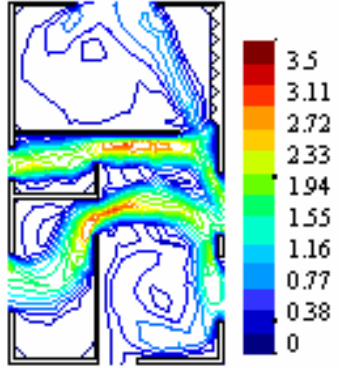

(b) Contour lines.
Figure 9: Contour fill and contour lines of $|\mathbf{u}|$ for Plan 2 and $\mathbf{u}=(1,0)$.
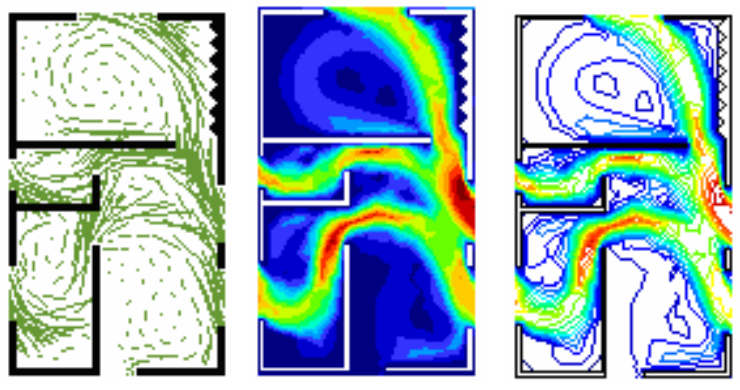

(a)Velocity field.
(b)Contour fill.

(c)Contour lines.
Figure 10: Velocity field, contour fill and contour lines of $|\mathbf{u}|$ for Plan 2 and $\mathbf{u}=(1,-1)$.
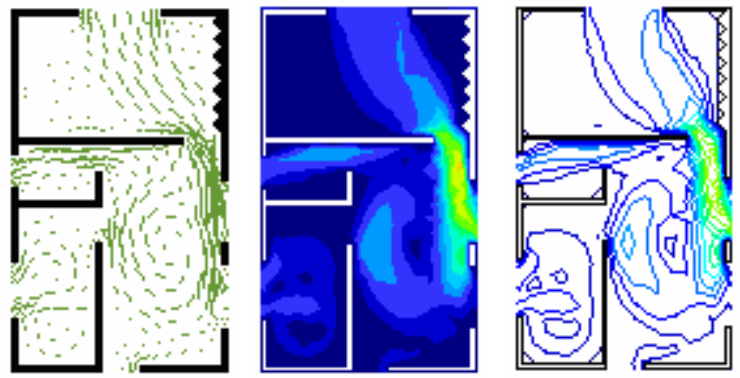

(a)Velocity field. (b)Contour fill. (c)Contour lines.

Figure 11: Velocity field, contour fill and contour lines of $|\mathbf{u}|$ for Plan 2 and $\mathbf{u}=(0,-1)$.

\section{Results for configuration Case 3 .}

Figures 12, 13, 14 and 15 present internal circulation results for the three given outside wind cases for Plan 3. The mesh has 7050 triangular elements and 3723 nodes as in Fig. 12(a). It can be observed an increase in the internal wind field intensity with circulation reaching a wider area when compared with the results for Plan 1 and Plan 2.

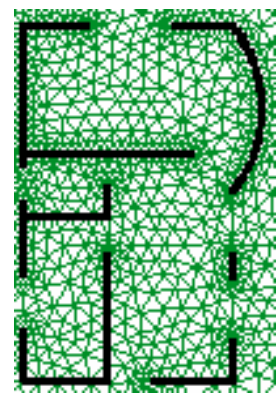

(a) Mesh detail.

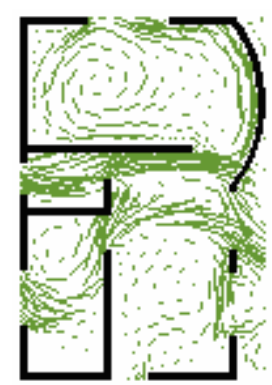

(b) Velocity field.
Figure 12: Mesh detail and wind field for Plan 3 and $\mathbf{u}=(1,0)$.

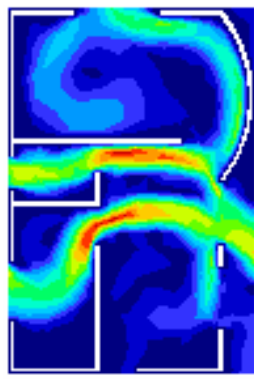

(a) Contour fill.

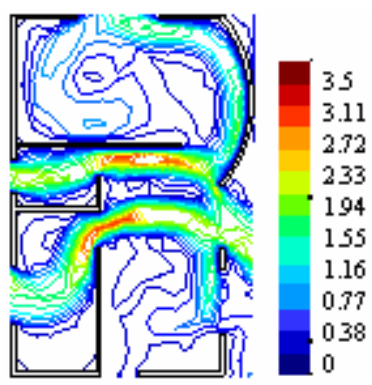

(b) Contour lines.
Figure 13: Contour fill and contour lines of $|\mathbf{u}|$ for Plan 3 and $\mathbf{u}=(1,0)$. 


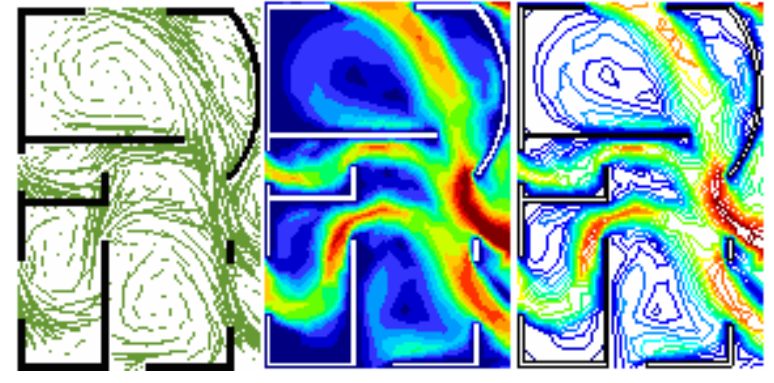

(a)Velocity field. (b)Contour fill. (c)Contour lines.

Figure 14: Velocity field, contour fill and contour lines of $|\mathbf{u}|$ for Plan 3 and $\mathbf{u}=(1,-1)$.

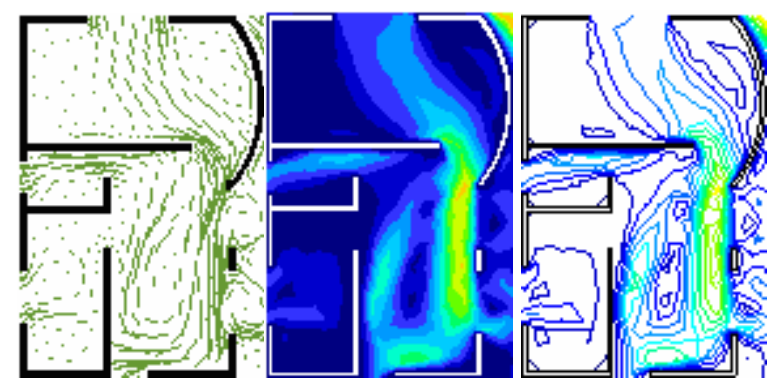

(a)Velocity field. (b)Contour fill. (c)Contour lines.

Figure 15: Velocity field, contour fill and contour lines of $|\mathbf{u}|$ for Plan 3 and $\mathbf{u}=(0,-1)$.

\section{Results for configuration Case 4 .}

Figures 16, 17, 18 and 19 present internal circulation results for the three given outside wind cases for Plan 4. The mesh has 7143 triangular elements and 3800 nodes as in Fig. 16(a). From the results we can observe that there is a decrease of the wind intensity when compared with the three other cases.

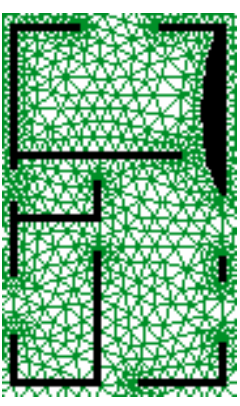

(a) Mesh detail.

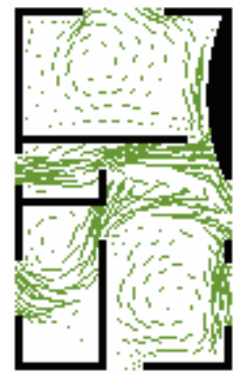

(b)Velocity field.
Figure 16: Mesh detail and wind field for Plan 4 and $\mathbf{u}=(1,0)$.

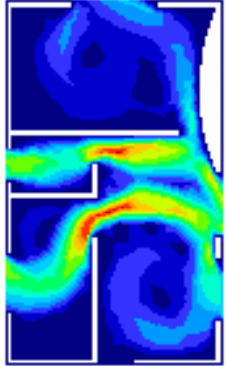

(a) Contour fill.

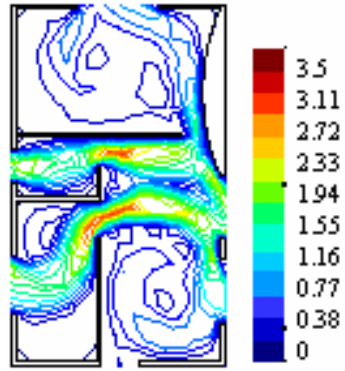

(b) Contour lines.
Figure 17: Contour fill and Contour lines of $|\mathbf{u}|$ for Plan 4 and $\mathbf{u}=(1,0)$.
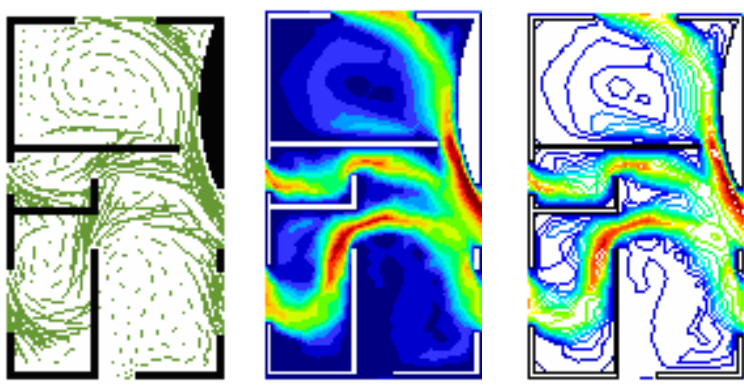

(a)Velocity field. (b)Contour fill. (c)Contour lines.

Figure 18: Velocity field, contour fill and contour lines of $|\mathbf{u}|$ for Plan 4 and $\mathbf{u}=(1,-1)$.
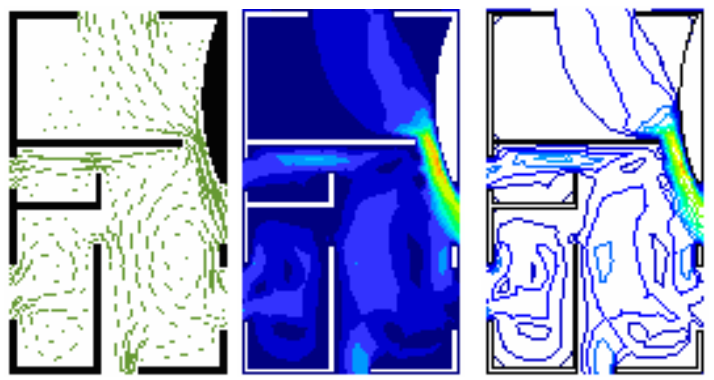

(a)Velocity field. (b)Contour fill. (c)Contour lines.

Figure 19: Velocity field, contour fill and contour lines of $|\mathbf{u}|$ for Plan 4 and $\mathbf{u}=(0,-1)$.

Table 1 presents a comparison for all the plans. Plan 1 has been taken as the reference configuration and it was attributed zero as the reference value, in a scale of $\{-1,0,1\}$. Value 1 was attributed to the case in which the ventilation increases and -1 was associated with the case in which ventilation decreases and the results for all the rooms have been obtained by summing the associated value of this scale to each of the winds. 
Table 1: Evaluation of Air Circulation for all Plans

\begin{tabular}{|l|c|c|c|c||c|}
\hline $\begin{array}{l}\text { Room } \\
\text { Plan } \downarrow\end{array}$ & $\begin{array}{c}\text { Room } \\
1\end{array}$ & $\begin{array}{c}\text { Room } \\
2\end{array}$ & $\begin{array}{c}\text { Room } \\
3\end{array}$ & $\begin{array}{c}\text { Room } \\
4\end{array}$ & $\sum$ \\
\hline Plan 1 & 0 & 0 & 0 & 0 & 0 \\
\hline Plan 2 & +3 & +1 & +1 & -1 & +4 \\
\hline Plan 3 & +3 & +1 & +1 & +1 & +6 \\
\hline Plan 4 & -1 & +1 & +1 & -3 & -2 \\
\hline
\end{tabular}

From Tab. (1) we could select the best ventilation cases, Plan 2 and Plan 3. Then, selecting one of them as the new reference, Plan 2 for instance, we could decide for the best alternative plan following Table (2); that is, Plan 3.

Table 2: Evaluation of Air Circulation for Plan 2 and Plan 3

\begin{tabular}{|l|c|c|c|c||c|}
\hline $\begin{array}{l}\text { Room } \\
\text { Plan } \downarrow\end{array}$ & $\begin{array}{c}\text { Room } \\
1\end{array}$ & $\begin{array}{c}\text { Room } \\
2\end{array}$ & $\begin{array}{c}\text { Room } \\
3\end{array}$ & $\begin{array}{c}\text { Room } \\
4\end{array}$ & $\sum$ \\
\hline Plan 2 & 0 & 0 & 0 & 0 & 0 \\
\hline Plan 3 & +3 & 0 & -1 & +1 & +4 \\
\hline
\end{tabular}

The criterion used to estimate the global circulation increase was to compare among the plans the following global circulation index $\left(\mathrm{K}_{\mathrm{D}}\right)_{\mathrm{G}}$ defined for each plan D as

$$
\left(K_{D}\right)_{G}=\sum_{j=1}^{N W}\left(\sum_{i=1}^{N V} \frac{(N V)_{i}}{A_{i}}\right)_{j}
$$

where (NV)i is the number of nodes of room $\mathrm{i}$ with absolute velocities higher than a reference value $\mathrm{Vr}$, $\mathrm{Ai}$ is the area of room $\mathrm{i}, \mathrm{j}$ refers to the wind case, $\mathrm{NW}$ is the total number of wind cases and NR is the total number of rooms in the plan.

With this criterion, Plan 3 presented the greater increase in the circulation, reaching of about $40 \%$ in relation to the original Plan 1 when $\mathrm{Vr}=0.3 \mathrm{~m} / \mathrm{s}$, and of about $30 \%$ when $\mathrm{Vr}=1.1 \mathrm{~m} / \mathrm{s}$ was considered. These values are inspiring to consider the shape of the walls in studying the distribution and intensity of indoor air circulation problems.

\section{CONCLUSION AND REMARKS}

In this work indoor air circulation in a specific built environment has been analyzed, with the objective of improving the ventilation of a plan, and consequently the thermal comfort, by a simple modification of the shape of one of the walls; seeking for an economic solution to the situation.

The analysis has been carried out by a PetrovGalerkin finite element method. Three external wind conditions have been considered.

By comparing the intensity of the velocities and the width of the strips in the velocity fill graphics, scaling $\{-1,0,1\}$ for $\{$ worst, reference, better $\}$ nd eliminating the worst cases successively we could decide for the best wall configuration to produce the best comfort state.

To confirm this selection we proposed another criterion based on the ratio of the number of elements with velocities above a reference value to each room area. In conclusion Plan 3 was the best configuration, presenting an increase of about $40 \backslash \%$ in ventilation in relation to the original Plan 1 when the reference velocity was adopted as $.3 \mathrm{~m} / \mathrm{s}$ and an increase of about $30 \%$ when the reference velocity was taken as $1.1 \mathrm{~m} / \mathrm{s}$.

\section{ACKNOWLEDGEMENTS}

Patricia R. C. Drach, would like to thank the support of FAPERJ - Fundação Carlos Chagas Filho de Amparo à Pesquisa do Estado do Rio de Janeiro and of the National Laboratory for Scientific Computing, LNCC/MCT.

José Karam F. would like to thank the support of the project PRONEX/FAPERJ no. E26/171.199/2003.

\section{REFERENCES}

Bragger, G. S., Dear, R. J., 2000, A Standard for Natural Ventilation, Ashrae Journal, Vol. 42, pp. 2128.

Brooks, A., Hughes, T J R., 1982. Streamline Upwind/Petrov-Galerkin formulations for convection dominated flows with particular emphasis on the incompressible Navier-Stokes equations, Computer Methods in Applied Mechanics Engineering. Vol. 32, pp 199-259.

Franca, L. P., Frey, S. L., 1992, Stabilized finite element methods: II. The incompressible NavierStokes equations, Computer Methods in Applied Mechanics Engineering, Vol. 99, pp. 209-233.

Galeão, A. C., do Carmo, E. G. D., 1988, A Consistent Approximate Upwind Petrv-Galerkin Methods for Convective Dominated Problems, Computer Methods in Applied Mechanics Engineering, Vol. 68, pp. 83-95.

Karam F., J., Loula, A. F. D., 1992, On Stable Equal-Order Finite Element Formulations for Incompressible Flow Problems, Int. J. Num. Meth. Engrg, Vol. 34, No. 2, pp. 655-665.

Szokolay, S. C., 1999, Approaches to Tropical House Design, in: Proceedings of the II Encontro Latino Americano de Conforto no Ambiente Construído, Vol. 1, Fortaleza, Brazil. 